\title{
Steigende Punitivität in der Bevölkerung ein Mythos?
}

\author{
Änderungen im Kriminalitätserleben der Bundesbürger und ihre Forderung \\ nach härteren Strafen
}

Karl- Heinz Reuband

\section{Einleitung}

S eit neustem ist, auch im kriminologischen Diskurs, wieder häufiger von steigendem Unsicherheitsgefühl und vom Strafverlangen der Bürger die Rede. Aber anders als früher als die polizeiliche Kriminalitätsstatistik noch einen Anstieg in der Bedrohung zu belegen schien - wird die Veränderung im Erleben nicht mehr auf Veränderungen in der objektiven Bedrohungslage zurückgeführt, sondern auf andere Faktoren. In einer Zeit, in der die Kriminalitätsrate rückläufig ist, fällt es schwer, diese als eine bedeutsame Einflussgröße für die Furchtentwicklung und steigendes Sanktionsverlangen anzusehen.

Für einige Autoren sind es vor allem gesellschaftliche Umbruchs- und Individualisierungsprozesse, die Menschen aus ihren etablierten sozialen Zusammenhängen lösen und sie in ihrer Gewissheit erschüttern. Zunehmende Verunsicherung und Angst würden ein steigendes Strafbedürfnis fördern (vgl. u.a. Niggli 2004). Für andere ist es die Ausbreitung des privaten, nicht öffentlich-rechtlichen Fernsehens, die eine vermehrte Berichterstattung über Kriminalität und Gewalt in Fernsehen bedingt und die Ausbreitung einer verzerrten Wahrnehmung der Kriminalitätslage begünstigt. Steigendes Strafverlangen wäre die Folge (Pfeiffer et al. 2004). Und für wieder andere sind es vor allem die Politiker, die durch eine wiederholte Thematisierung von Kriminalität in der Bevölkerung Verunsicherung hervorrufen und ein punitives Meinungsklima schaffen (vgl. u.a. Kreissl und Fischer 2003:115; Christie 2005).

Wie auch immer die Entwicklung des Sicherheitsgefühls und dessen Ursachen eingeschätzt werden mag, für eine ganze Reihe von Autoren gilt es inzwischen als gesichert, dass die Punitivität in der Gesellschaft in den letzten Jahren zugenommen hat. Die Rede ist von einer „selbstverständlichen Strafbereitschaft, ja Straffreude“, die sich ausgebreitet hätte (Hassemer 2001) von neuer "Straflust", „von einem Punitivitätsschub“ (Lautmann und Klimke 2005:17). Mehrere Autoren meinen sogar, dass sich die Bundesrepublik in dieser Hinsicht inzwischen den punitiven Verhältnissen wie sie für die USA und Großbritan- nien beschrieben sind (vgl. Garland 2001) angenährt hätte. Und nicht wenige meinen, dass sich diese Entwicklung auch in der Zukunft fortsetze (vgl. u.a. Peters und Sack 2003: 25).

So berechtigt die Diagnose eines punitiven Wandels in der Beschreibung der Strafgesetzgebung und Rechtspraxis womöglich auch sein mag, so sehr fragt sich andererseits jedoch, ob die Strafvorstellungen in der Bevölkerung tatsächlich punitiver geworden sind. Die bisherigen Arbeiten, in denen dies unter Bezug auf empirische Befunde behauptet wurde, erweisen sich bei näherer Betrachtung als wenig überzeugend. So sagt die wiederholt als Beleg zitierte Untersuchung von Heinz Streng (2000), in der Änderungen des Strafverlangens unter Erlanger Jurastudenten dokumentiert werden, letztlich nur etwas über diese Population aus, nicht über die Bevölkerung als Ganzes. Desgleichen lässt die Untersuchung von Christian Pfeiffer und Mitarbeitern, in der eine Beziehung zwischen Mediennutzung, Einschätzung der Kriminalitätslage und Strafverlangen ermittelt wurde (Pfeiffer et al. 2004), nur Aussagen auf individueller Ebene zum jeweiligen Erhebungszeitpunkt zu, nicht aber über gesamtgesellschaftliche Trends.

Pfeiffer und Mitarbeiter meinen zwar, aus den Befunden ihrer Querschnittsuntersuchung Schlüsse für längerfristige Änderungen ableiten zu können: schließlich sei die Nutzung der privaten Sender gestiegen und Kriminalitätsdarstellung hätten dadurch im Fernsehen an Bedeutung gewonnen. Doch ob die konstatierten Beziehungen auf Individualebene ausreichen, um einen Wandel auf der Makroebene zu bewirken, ist angesichts konkurrierender Einflusse keineswegs sicher. Auch wenn es einen Medieneffekt geben sollte, ist er doch im Vergleich zu den anderen Einflussfaktoren womöglich derart gering, dass sich daraus keine nennenswerten Auswirkungen auf der Makroebene ergeben.

Die spärlichen empirischen Untersuchungen, die Vergleiche über die Zeit erlauben, wecken Zweifel an der These steigender Punitivität. So erbrachte ein Vergleich des Strafverlangens für einige ausgewählte Delikte für die Jahre 1970, 1982, 1987 und 2003 keine einheitliche Entwicklung. Steigendes Strafverlangen zeichnete nur ei- nes der Delikte aus - Gewalt gegen die Ehefrau. Bei den anderen Delikten herrschten konstante Verhältnisse oder sogar ein rückläufiges Strafverlangen vor (Reuband 2004). Ebenso wenig konnte ein Vergleich der Jahre 1989 und 2002 ein steigendes Strafverlangen für das Delikt Einbruch ermitteln. Allenfalls in der jüngsten Alterskohorte deuteten sich - wenn auch nur schwach - Tendenzen in diese Richtung an (Reuband 2003).

In der öffentlichen Debatte gilt seit Jahren als ausgemacht, dass die populären Medien eine diffuse Verbrechensfurcht anheizen. Ob aber das allgemeine Unsicherheitsgefühl immer noch diese Folgen hat, wird zunehmend fraglich. Zwar ist es richtig, dass gesellschaftliche Umbruchs- und Individualisierungsprozesse die Menschen aus ihren etablierten sozialen Zusammenhängen lösen, aber sie haben deshalb kein steigendes Strafbedürfnis mehr, sondern gewöhnen sich an die oft mit Kriminalität uns Unordnung assoziierte Spätmoderne. Jedenfalls sind seit einigen Jahren die Mehrheit der deutschen Bürger- und Bürgerinnen der Ansicht, dass die bestehenden Strafgesetze durchaus ausreichen, um den sich stellenden Problemen gerecht zu werden. Kriminalität ist also nicht mehr die Chiffre für die aktuellen Ängste in unserer Gesellschaft. Konjunktur haben vielmehr die Angst vor dem drohenden Arbeitsplatzverlust und Risiken des weltweit praktizierten Terrorismus.

Die einzige Trenduntersuchung, die auf eine steigende Punitivität hindeutet, stammt vom Kriminologischen Forschungsinstitut Niedersachen. Basis sind zwei Umfragen aus den Jahren 1992 und 2004. Die Ergebnisse des Vergleichs finden sich in einer Publikation von Christian Pfeiffer (Pfeiffer et al. 2004) beiläufig, mehr am Rande erwähnt. Danach ist, gemessen am Mittelwert der Skala, die Zustimmung zum Statement "Harte Strafen sind notwendig, damit andere davon abgehalten werden, Straftaten zu begehen" gestiegen. Pfeiffer schreibt, es handele sich bei dem Statement um ein Beispiel aus einer umfassenderen Batterie von Statements. Und diese würden in ihrer Gesamtheit steigende Punitivität belegen. Er unterlässt es aber leider, die anderen Statements $\mathrm{zu}$ benennen und ihre Entwicklung im einzelnen 
zu dokumentieren. Ob das zitierte „Beispiel“ in besonders eindrucksvoller Weise den Trend wiedergibt und deshalb gewählt wurde oder ob andere Gründe zu seiner Auswahl führten, wird nicht erwähnt. Offen bleibt ebenso, ob sich die Angaben allein auf Westdeutschland oder auf Gesamtdeutschland beziehen. Und dies ist keinesfalls trivial: Die Entwicklungen könnten angesichts unterschiedlicher Ausgangslagen durchaus verschiedene sein und bei Zusammenfassung zu Gesamtdeutschland sich unter Umständen gar ausgleichen.

\section{Zielsetzung und methodisches Vorgehen}

Im Folgenden soll auf der Basis einer jüngst erhobenen Umfrage des Instituts für Demoskopie Allensbach und unter Rückgriff auf frühere Erhebungen ein Zeitvergleich punitiver Orientierungen in der deutschen Bevölkerung unternommen werden. Grundlage sind bundesweite, repräsentative Erhebungen der Bevölkerung 16 Jahre und älter. Die Erhebungen wurden in der Regel face-to-face durchgeführt und stützen sich auf eine Quotenstichprobe. Anders als in den Umfragen, in denen die Bewertung einzelner Delikte erfragt ist, wurde ein generalisiertes, deliktunabhängiges Strafverlangen ermittelt. Dies erfolgte zum einen über eine Frage zur Abschreckungswirkung harter Strafen („Wenn man Verbrechen härter bestrafen würde, gäbe es dann weniger Verbrechen, oder glauben Sie das nicht?"), zum anderen über eine Frage zum Sinn von Strafverschärfungen („Was würden Sie sagen, brauchen wir bei uns in Deutschland schärfere Gesetze gegen Kriminalität, oder halten Sie die Gesetze, die wir haben, für ausreichend?").

Ausgewählte Ergebnisse aus diesem Vergleich wurden für Gesamtdeutschland von Elisabeth Noelle und Wolfgang Petersen unlängst in der „Frankfurter Allgemeinen Zeitung" publiziert (2006). Hier nun soll unter Rückgriff auf diese und andere Daten (die in Tabellenform dankenswerterweise vom Allensbacher Institut zur Verfügung gestellt wurden) eine vertiefende Analyse, unter besonderer Berücksichtigung der Verhältnisse in West- und Ostdeutschland, unternommen werden. In diesem Zusammenhang soll auch eine Zeitreihe zum Sicherheitsgefühl der Bundesbürger, die wir 1999 in dieser Zeitschrift publizierten (Reuband 1999) und die sich ebenfalls auf Allensbacher Daten stützt, aktualisiert und in die Diskussion einbezogen werden. Dadurch wird es möglich, die Veränderungen punitiver Einstellungen vor dem Hintergrund der Entwicklungen im Bereich des Sicherheitsgefühls zu interpretieren.

\section{3. Änderungen im subjektiven Kriminalitätserleben}

Wie sich die Kriminalitätsfurcht der Bürger seit den 70 Jahren entwickelt hat, ist in Tabelle $1 \mathrm{zu}$ sammengestellt. Die Zeitreihe weist zwar für bestimmte Zeiträume Lücken auf - besonders für die zweite Hälfte der 80er Jahre. Man kann diese Lücken jedoch durch andere Zeitreihen füllen (vgl. Reuband 1995). Das Gesamtbild, das sich zusammen mit diesen anderen Quellen darbietet, spricht für einen Rückgang der Kriminalitätsfurcht in Westdeutschland bis Ende der 80er Jahre. Mit der Wiedervereinigung kommt es dann zu einer Zäsur: der Rückgang der Kriminalitätsfurcht setzt sich nicht mehr fort, stattdessen setzt ein dramatischer Anstieg der Furcht ein. Dieser Anstieg erreicht seinen Höhepunkt 1993, weicht in den Folgejahren aber dann wieder einem Rückgang. Dieser ist inzwischen in eine Phase weitgehender Stabilität übergegangen. Die Entwicklung hat sich auf einem weitaus niedrigeren Niveau als noch Anfang der 90er Jahre eingependelt. Das Niveau der 70er Jahre ist freilich in Westdeutschland noch nicht wieder erreicht.

Sowohl der jähe Anstieg zu Beginn der 90er Jahre als auch der Rückgang in der Folgezeit kennzeichnet die Entwicklung sowohl in Westwie auch Ostdeutschland. Auffällig dabei ist, dass sich die Ostdeutschen von Anfang der Beobachtungsperiode an durch ein weitaus höheres Maß an Beunruhigung auszeichneten als die Westdeutschen, sich die Verhältnisse dann aber bis Ende der 90er Jahre weitgehend anglichen. Mangelnde Vertrautheit mit Kriminalität als Massenphänomen, seltenere Medienberichterstattung

Tabelle 1: Kriminalitätsfurcht im Zeitverlauf (in \%)

\begin{tabular}{|c|c|c|c|c|c|c|c|c|c|c|c|c|c|c|c|}
\hline & \multicolumn{11}{|c|}{ Westdeutschland } & \multicolumn{4}{|c|}{ Ostdeutschland } \\
\hline & 75 & 79 & 80 & 82 & 91 & 92 & 93 & 94 & 99 & 01 & 04 & 92 & 93 & 94 & 99 \\
\hline $\begin{array}{l}\text { Dass bei mir zu Hause } \\
\text { eingebrochen wird }\end{array}$ & 21 & 20 & 21 & 21 & 57 & 43 & 41 & 40 & 32 & 24 & 26 & 68 & 59 & 59 & 31 \\
\hline $\begin{array}{l}\text { Dass ich überfallen und } \\
\text { beraubt werde }\end{array}$ & 20 & 17 & 18 & 23 & - & 38 & 35 & 27 & 30 & 26 & 27 & 60 & 55 & 52 & 31 \\
\hline $\begin{array}{l}\text { Dass ich unterwegs } \\
\text { bestohlen werde }\end{array}$ & 12 & 9 & 14 & 15 & - & 29 & 30 & 24 & 22 & 18 & 22 & 51 & 45 & 42 & \\
\hline $\begin{array}{l}\text { Dass ich von einem } \\
\text { Betrüger um meine } \\
\text { Ersparnisse gebracht werde }\end{array}$ & 7 & 9 & 8 & - & - & 13 & 6 & 10 & 8 & 8 & 11 & 28 & 17 & 20 & 11 \\
\hline $\begin{array}{l}\text { Dass mich aggressive } \\
\text { Jugendlichen (Skinheads, } \\
\text { Punks) auf der Straße } \\
\text { angreifen } \\
\text { Dass jemand aus meiner } \\
\text { Familie oder gute Freunde } \\
\text { Opfer eines Verbrechens } \\
\text { werden }\end{array}$ & - & - & - & - & 44 & - & 37 & 34 & 34 & 28 & 33 & - & 45 & 51 & 37 \\
\hline
\end{tabular}

- nicht erfragt

Quellen: Veröffentlichte und unveröffentlichte Umfragen des Instituts für Demoskopie (vgl. auch Reuband 1999). Wo mehrere Werte pro existieren, wurde ein Durchschnittswert errechnet. Frageformulierung: „Kommt es in letzter Zeit vor, dass Sie Angst haben vor etwas, was auf dieser Liste steht?“ 
im Vergleich der Jahre 1993 und 2006 bei den Westdeutschen, wie man Tabelle 2 entnehmen kann, kein nennenswerter Anstieg im Glauben an eine abschreckende Wirkung. Die Zahl derer, die daran glauben, ist nur geringfügig um vier Prozentpunkte gestiegen. Zugleich ist die Zahl der Unentschiedenen leicht gestiegen und die Zahl der Zweifler zurückgegangen. Setzt man die Angaben für Glaube und Nichtglaube an die abschreckende Wirkung in Relation zueinander, so hat sich das Meinungsverhältnis leicht zugunsten eines Glaubens an die Abschreckungswirkung verschoben. Alles in allem jedoch ist diese Änderung relativ schwach. Unter den Ostdeutschen, die 1993 von einem weitaus höheren Niveau der Akzeptanz der Abschreckungsdoktrin ausgingen als die Westdeutschen, hat es im Zeitverlauf hingegen einen sehr deutlichen Rückgang im Glauben an die Abschreckungsfunktion gegeben: von $64 \%$ auf $55 \%$.

Nun ist der Glaube an die Abschreckungswirkung von Strafen, genau genommen, nur im erzwar ist der Glaube daran in der Regel ein Bestandteil punitiven Denkens. Doch muss jemand, der harte Strafen für abschreckend hält, nicht notwendigerweise auch der Ansicht sein, man müsse die Strafandrohung erhöhen. Er könnte, solange er alternative „mildere“ Reaktionen für wirksam oder moralisch für legitimierbar hält, sich ebenso für diese aussprechen und für wohlfahrtstaatliche Maßnahmen plädieren.

Ein besserer Indikator für punitives Denken wird in den vorliegenden Umfragen des Instituts für Demoskopie über die Frage erfasst, ob man in Deutschland schärfere Gesetze gegen die Kriminalität benötige. Der Vergleich der Antworten dokumentiert - wie man Tabelle 3 entnehmen kann - in Westdeutschland im Anteil der Befürworter schärferer Gesetze eine weitgehende Konstanz weiterten Sinne ein Indikator für Punitivität:

Abbildung: Wahrgenommene persönliche Bedrohung durch Kriminalität im Zeitverlauf (in \%)

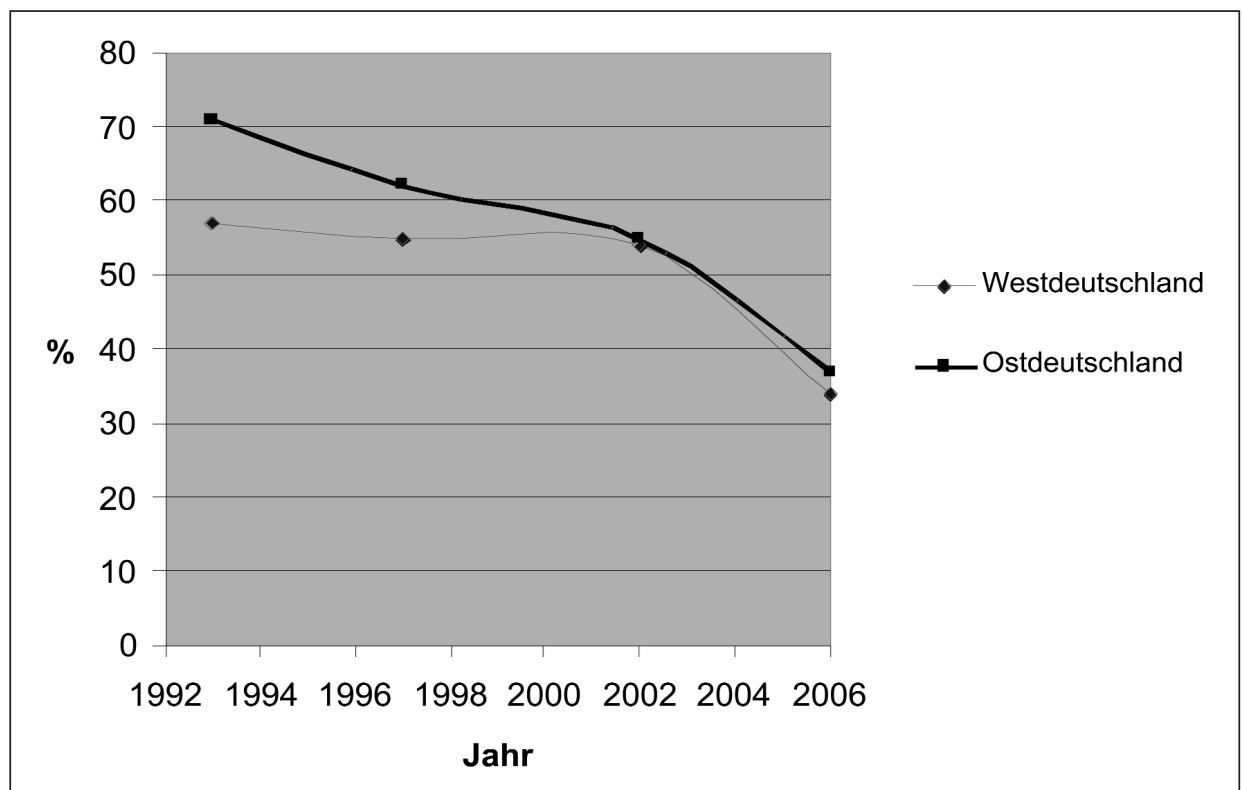

Aufgeführt sind Personen, die sich „sehr“ oder „etwas“ durch Kriminalität bedroht fühlen. Der verbleibende Anteil besteht aus Personen, die sich nicht bedroht fühlen oder unentschieden sind.

Frageformulierung 1993 und 1997: „Haben Sie Angst, es könnte Ihnen etwas passieren, Sie könnten Opfer eines Verbrechens werden? Wie sehr fühlen Sie sich durch Verbrechen bedroht? Würden Sie sagen, Sie fühlen sich..." Antwortkategorien: „sehr bedroht - etwas bedroht - nicht bedroht - Unentschieden“; Frageformulierung 2002 und 2006: „Manche Menschen haben ja Angst, es könnte ihnen etwas passieren, sie könnten Opfer eines Verbrechens werden. Wie sehr fühlen Sie sich durch Verbrechen bedroht? Würden Sie sagen, Sie fühlen sich ..." Antwortkategorien: „sehr bedroht - etwas bedroht - nicht bedroht - Unentschieden“ der Verhältnisse zwischen 1998 und 2003 und in der Folgezeit dann einen Rückgang in der Befürwortung. Würde man sich auf die Befragten beschränken, die eine inhaltliche Position gegenüber der Frage einnehmen und die Kategorie „Unentschieden“ aus der Berechnung ausklammern, wäre bereits zwischen 1998 und 2003 ein
Tabelle 2: Wahrgenommen Wirkung harter Strafen im Zeitverlauf (in \%)

\begin{tabular}{|c|c|c|c|c|}
\hline & \multicolumn{2}{|c|}{ Westdeutschland } & \multicolumn{2}{|c|}{ Ostdeutschland } \\
\hline & 1993 & 2006 & 1993 & 2006 \\
\hline $\begin{array}{l}\text { Weniger } \\
\text { Verbrechen }\end{array}$ & 46 & 50 & 64 & 55 \\
\hline Ohne Wirkung & 43 & 35 & 26 & 35 \\
\hline Unentschieden & 11 & 15 & 10 & 10 \\
\hline & 100 & 100 & 100 & 100 \\
\hline
\end{tabular}

Frageformulierung: „Wenn man Verbrechen härter bestrafen würde, gäbe es dann weniger Verbrechen, oder glauben Sie das nicht?“
Rückgang eingetreten. ${ }^{1}$ In Ostdeutschland ist die Entwicklung nicht minder eindrucksvoll: der Anteil, der sich für ein punitiveres Vorgehen ausspricht, geht hier von $76 \%$ auf $67 \%$ zurück.

Auffällig am Vergleich der west- und ostdeutschen Angaben ist es, dass sich die Bewohner der beiden Landesteile bei der Frage nach der Wirkung harter Strafen heutzutage nicht mehr unterscheiden, wohl aber in ihrem Ruf nach schärferen Gesetzen. Ein Anteil von $49 \%$ auf Seiten der Westdeutschen steht einem Anteil von $67 \%$ auf Seiten der Ostdeutschen gegenüber. Dies ist auch deswegen bemerkenswert, weil das Sicherheitsgefühl in West- und Ostdeutschland inzwischen nahezu identisch ist und frühere Untersuchungen - darunter auch die Erhebung zum Strafverlangen bei einem Einbruchsdelikt (vgl. Reuband 2003) - keine größeren Unterschiede zwischen West- und Ostdeutschen erbrachten. ${ }^{2}$

Ob sich in der häufigeren Befürwortung schärferer Gesetze unter den Ostdeutschen die Restbestände einstiger autoritärer Verhältnisse zu DDR Zeiten oder neue Unsicherheiten widerspiegeln, ist ungewiss. Sicher ist: auch wenn man die Kriminalitätsfurcht als eine mögliche Determinante des Sanktionsverlangens berücksichtigt, bleibt der Unterschied zwischen West- und Ostdeutschen bestehen: Sowohl unter denen, die sich 
durch Kriminalität beunruhigt fühlen wie auch unter denen, die sich sicher fühlen, plädieren mehr Ost- als Westdeutsche für ein härteres Durchgreifen. ${ }^{3}$ Die Erklärung der Unterschiede kann somit nicht darauf zurückgeführt werden, dass Ostdeutsche stärker als Westdeutsche auf wahrgenommene Bedrohung mit einem Ruf nach Strafverschärfung reagieren.

Auch ergeben sich keine Anzeichen dafür, dass der Ost-West-Unterschied eine Folge unterschiedlich intensiver Mediennutzung sein könnte. Zwar nutzen die Ostdeutschen das Fernsehen etwas intensiver als die Westdeutschen - überproportional auch die privaten Fernsehsender (Zubayr und Gerhard 2006:126) -, und müssten, folgt man Pfeiffers Argumentation, daher häufiger Sendungen mit Kriminalitätsinhalten rezipieren. Aber die Ost-West- Unterschiede sind nicht ein Resultat dessen. Denn selbst wenn man die Dauer des Fernsehens berücksichtigt, bleiben sowohl unter denen mit wenig als auch mit viel Fernsehkonsum die Ostdeutschen punitiver eingestellt als die Westdeutschen. ${ }^{4}$

\section{Die soziale Basis punitiver Orientierungen}

Welche sozialen Gruppen sind es nun, in denen man den Ruf nach schärferen Gesetzen überproportional oft finden kann? Anders als man es zunächst aufgrund früherer Literatur vermuten könnte, erweisen sich Frauen im Vergleich zu den Männern nicht als weniger, sondern als stärker punitiv. Ihre übliche Neigung, sich für ein milderes Strafmaß auszusprechen und harte Strafen wie z.B. die Todesstrafe - abzulehnen, schlägt sich nicht in entsprechenden Antworten nieder. Möglicherweise wirkt sich hier aus, dass in den letzten Jahren gehäuft spektakuläre Sexualdelikte gegen Kinder zum Gegenstand der Medienberichterstattung wurden und Frauen in erster Linie an

Tabelle 3: Forderung nach schärferen Gesetzen im Zeitverlauf (in \%)

\begin{tabular}{|l|c|c|c|c|c|c|}
\hline & \multicolumn{3}{|c|}{ Westdeutschland } & & \multicolumn{3}{|c|}{ Ostdeutschland } \\
\cline { 2 - 3 } \cline { 6 - 7 } & 1998 & 2003 & 2006 & 1998 & 2003 & 2006 \\
\hline $\begin{array}{l}\text { Schärfere } \\
\text { Gesetze }\end{array}$ & 64 & 64 & 49 & 76 & 69 & 67 \\
\hline $\begin{array}{l}\text { Gesetze } \\
\text { ausreichend }\end{array}$ & 22 & 31 & 42 & 14 & 23 & 25 \\
\hline Unentschieden & 14 & 5 & 9 & 10 & 8 & 8 \\
\hline & 100 & 100 & 100 & 100 & 100 & 100 \\
\hline
\end{tabular}

Frageformulierung: „Was würden Sie sagen, brauchen wir bei uns in Deutschland schärfere Gesetze gegen die Kriminalität, oder halten Sie die Gesetze, die wir haben, für ausreichend?" derartige Delikte denken, wenn sie sich für schärfere Gesetze aussprechen.

Was das Alter betrifft, so sind es überproportional die Älteren, die sich für eine Gesetzesverschärfung aussprechen. Die Beziehung ist nicht sonderlich stark ausgeprägt (lediglich die über 60jährigen weichen markanter $a b$ ), aber sie ist konsistent mit früheren Befunden über Strafeinstellungen. Wie sehr es sich um reine Alters- oder Kohorteneffekte handelt, kann hier nicht beantwortet werden. Im Fall der Todesstrafe, die mit dem Grundgesetz abgeschafft wurde, war noch in Ansätzen ein Kohorteneffekt nachzuweisen (Reuband 1975). Beim Ruf nach schärferen Gesetzen könnte jedoch auch eine altersbedingte „Vulnerabilität" gegenüber Kriminalität - die auch für erhöhte Kriminalitätsfurcht unter Älteren mitverantwortlich ist (Reuband 1999) - eine Rolle spielen.

Schließlich zeigt sich im Fall der Bildung, was sich auch schon früher mehrfach in Untersuchungen gezeigt hat: die besser Gebildeten sind weniger punitiv eingestellt als die schlechter Gebildeten. Allerdings ist dies keine Beziehung, die man als quasi zwangsläufig ansehen kann. Wie sich bei einem Vergleich der Einstellung zur Todesstrafe gezeigt hat, repräsentieren in den 50er Jahren am stärksten zunächst Personen mit Gymnasialbildung die Befürworter der Todesstrafe. Erst in den 60er Jahren kehrte sich diese Beziehung um. Heutzutage findet sich die Anhängerschaft der Todesstrafe vor allem unter Personen mit Volks- bzw. Hauptschulbildung (Reuband 1990). Die häufig vertretene Vorstellung, besser Gebildete wären aufgrund ihren hohen kognitiven Kompetenz immun gegenüber einfacher Losung und Vorurteilen, verkennt, dass auch sie den Einflüssen der politischen Kultur ausgesetzt sind. Und dies aufgrund der längeren Schulzeit stärker als dies bei den schlechter Gebildeten der Fall ist. Dementsprechend waren in Generatio-

\section{Schlussbemerkungen}

Die Annahme, die Bundesbürger seien in den letzten Jahren punitiver geworden, scheint ein Mythos zu sein. Es gibt keine empirischen Befunde, die einen derartigen Trend belegen. Zwar hat sich, ähnlich wie es Pfeiffer et al. erwähnt hat (Pfeiffer et al. 2004), das Meinungsbild leicht zugunsten einer Befürwortung der Abschreckungswirkung harter Strafen verschoben (wenn man die Relation der jeweiligen Meinungen zueinander in Beziehung setzt bzw. Mittelwerte bildet). Doch eine Zunahme des Glaubens an die abschreckende Wirkung harter Strafen ist nicht notwendigerweise mit einer Zunahme punitiver Orientierungen identisch. Fasst man Punitivität stringenter und wählt das Verlangen nach Strafverschärfung als Massstab, so kann man in den letzten Jahren eher eine Abnahme der Punitivität verzeichnen.

Inwieweit zu dieser Entwicklung beigetragen hat, dass die Kriminalitätsfurcht seit 1993 sank, ist ungeklärt. Subjektive Bedrohtheitsgefühle müssen sich nicht, wie andere Forschung gezeigt hat, notwendigerweise in einem punitiven Strafverlangen niederschlagen. Aber sie können es. Unter welchen Bedingungen dies der Fall ist, bedarf weiterer Klärung. Eines jedoch ist sicher: je geringer das Gefühl der Bedrohung ist, desto schwerer fällt es, für „Moralunternehmen“ und Politiker darauf Bezug zu nehmen. Kriminalität ist unter diesen Umständen keine „kognitive Ressource", auf die der Konstruktion sozialer Bedrohung zurückgegriffen werden kann.

Mit unserem Ergebnis eines stabilen bzw. sinkenden Strafverlangens, wird die weitverbreitete These steigender Punitivität auf Seiten der Bundesbürger in Zweifel gezogen. Mag es auch 
Tabelle 4: Forderung nach schärferen Gesetzen in Abhängigkeit von Ost/Westzugehörigkeit und nach sozialen Merkmalen

\begin{tabular}{|c|c|c|c|c|c|c|c|c|}
\hline & \multicolumn{2}{|c|}{ Geschlecht } & \multicolumn{4}{|c|}{ Alter } & \multicolumn{2}{|c|}{ Bildung } \\
\hline & Mann & Frau & $\begin{array}{l}16- \\
29\end{array}$ & $\begin{array}{c}30- \\
44\end{array}$ & $\begin{array}{c}45- \\
59\end{array}$ & $60+$ & $\begin{array}{c}\text { Einfache } \\
\text { Schule }\end{array}$ & $\begin{array}{l}\text { Höhere } \\
\text { Schule }\end{array}$ \\
\hline \multicolumn{9}{|l|}{ Westdeutschland } \\
\hline Schärfere Gesetze & 44 & 53 & 42 & 48 & 46 & 55 & 51 & 46 \\
\hline $\begin{array}{l}\text { Gesetze } \\
\text { ausreichend }\end{array}$ & 49 & 36 & 45 & 42 & 45 & 39 & 41 & 44 \\
\hline Unentschieden & 7 & 11 & 13 & 10 & 9 & 6 & 8 & 11 \\
\hline \multicolumn{9}{|l|}{ Ostdeutschland } \\
\hline Schärfere Gesetze & 64 & 69 & 59 & 65 & 65 & 73 & 76 & 61 \\
\hline $\begin{array}{l}\text { Gesetze } \\
\text { ausreichend }\end{array}$ & 29 & 22 & 32 & 23 & 28 & 21 & 20 & 28 \\
\hline Unentschieden & 7 & 10 & 9 & 12 & 7 & 6 & 5 & 10 \\
\hline
\end{tabular}

Die Zahlen addieren sich jeweils in den Rubriken für West- und Ostdeutschland spaltenweise auf $100 \%$. Die Zahlen der Befragten pro Subgruppe variieren zwischen ca. 100 und 350 Personen.

Basis: Erhebung von 2006

den Anschein haben, als würden in der Öffentlichkeit mehr Stimmen als früher, bei spezifischen Delikten ein härteres Vorgehen fordern, ob ähnliche Entwicklungen in der Bevölkerung sich vollziehen und ob diese über die spezifischen Delikte hinaus generalisiert werden können, ist keineswegs sicher.

\section{Literatur:}

Christie, N.: Wieviel Kriminalität braucht die Gesellschaft?, München 2005

Garland, D.: The Culture of Control. Crime and Social Order in Contemporary Society. Oxford 2001

Hassemer, W.: Die neue Lust auf Strafe, in: Frankfurter Rundschau Nr. 296 vom 20.12.2001

Kreissl, R. und M. Fischer: European Folksdevils und die Politik Innerer Sicherheit in der Festung Europa, in: W. Stangl und G. Hanatz, Hg., Innere Sicherheiten. Baden-Baden 2003, S. 113-135

Lautmann, R. und D. Klimke: Punitivität als Schlüsselbegriff für kritische Kriminologie, in: R. Lautmann, D. Klimke und F.Sack, Hrsg, Punitivität. 8. Beiheft Kriminologisches Journal, 2004

Niggli, M.A.: Wie viel Strafe braucht der Mensch? Zur Strafrechtsdebatte in Europa, in: Stapferhaus Lenzburg, Hg., Strafen. Ein Buch zur Strafkultur der Gegenwart. Baden 2004, S. 184-199

Noelle-Neumann, E. und R. Köcher: Allensbacher Jahrbuch der Demoskopie 1984-1992. München 1993

Noelle-Neumann, E. und R. Köcher: Allensbacher Jahrbuch der Demoskopie 1993-1997, München 1997

Noelle, E. und T. Petersen: Wie sicher ist Deutschland? Die Furcht der Bürger vor Verbrechen nimmt ab, in:
Frankfurter Allgemeine Zeitung: 10.07. 2006, S. 5

Peters, H. und F. Sack: Von mäßiger Fortschrittlichkeit und soziologischer Ignoranz, in: Kriminologisches Journal 35, 2003, S. 17-29

Pfeiffer, C., M. Windzio und M. Kleimann: Die Medien, das Böse und wir. Zu den Auswirkungen der Mediennutzung auf Kriminalitätswahrnehmung, Strafbedürfnisse und Kriminalpolitik, in: Monatsschrift für Kriminologie und Strafrechtsreform, Heft 6, 2004, S. 415-435

Reuband, K.-H.: Die Einstellung zur Todesstrafe in der Bundesrepublik Deutschland 1960-1970. Ergebnisse einer Kohortenanalyse, in: Angewandte Sozialforschung, 1, 1975, S. 31-46

Reuband, K-H.: Sanktionsverlangen im Wandel. Die Einstellung zur Todesstrafe in der Bundesrepublik Deutschland seit 1950, in: Kölner Zeitschrift für Soziologie und Sozialpsychologie, 32, 1980, S. 535-558 Reuband, K.-H.: Kriminalitätsfurcht in Ost- und Westdeutschland. Zur Bedeutung psychosozialer Einflußfaktoren, in: Soziale Probleme, 3, 1992, S. 211-219

Reuband, K.-H.: Veränderungen in der Kriminalitätsfurcht der Bundesbürger. Eine Bestandsaufnahme empirischer Erhebungen, in: G. Kaiser und M. Jehle, Hrsg., Kriminologische Opferforschung. Neue Perspektiven und Erkenntnisse. Band II. Heidelberg 1995, S. 37-54

Reuband, K.-H., Konstanz und Wandel im Strafbedürfnis der Bundesbürger - 1970 bis 2003, in: R. Lautmann, D. Klimke und F.Sack, Hrsg, Punitivität. 8. Beiheft des Kriminologisches Journal, 2004, S. 89-103

Reuband, K.-H.: Kriminalitätsfurcht im Alter. Ergebnisse ostdeutscher Studien, in: K. Lenz, M. Rudolph und U. Sieckendiek, Hrsg., Die alternde Gesellschaft. Problemfelder gesellschaftlichen Umgangs mit Altern und Alter. München 1999, S. 209-231

Reuband, K.-H.: Von der Kriminalitätshysterie zur Normalität?, in: Neue Kriminalpolitik. Forum und Praxis, Politik und Wissenschaft 11, Heft 4, 1999, S. 16-19

Reuband, K.-H.: Steigende Repressionsneigung im Zeitalter der „Postmoderne“? Das Sanktionsverlangen der Bundesbürger 1989 und 2002 im Vergleich, in: Neue Kriminalpolitik, Heft 3, 2003, S. 100-104

Reuband, K.-H.: 2006 Das NS-Regime zwischen Akzep$\operatorname{tanz}$ und Ablehnung. Eine retrospektive Analyse von Bevölkerungseinstellungen im Dritten Reich auf der Basis von Umfragedaten, in: Geschichte und Gesellschaft. Zeitschrift für Historische Sozialwissenschaft, 31, 2006 (im Druck)

Streng, F.: Die heranwachsende Juristengeneration und die Aufgabe des Strafrechts, in: Bewährungshilfe, 4, 2000, S. 422-435

Zubayr, C. und H. Gerhard: Fernsehgewohnheiten und Fernsehreichweiten im Jahr 2005, in: Media Perspektiven 3/2006, S. 125-137

Der Verfasser ist erreichbar über das Sozialwissenschaftliche Institut der Heinrich- Heine Unversität Düsseldorf

\section{Fußnoten}

1 Klammert man die „Unentschiedenen“ aus, kommt man 1998 auf einen Wert von 74\% Befürwortung, 2003 von $67 \%$ und 2006 von 54\%

2 Allerdings gab es schon früher unter den Bürgern der neuen Länder ein Plädoyer für ein härteres Durchgreifen und schärfere Gesetze - nur war damals auch noch das Gefühl von Kriminalitätsbedrohung weiter verbreitet. So sprachen sich 199287 \% der Ostdeutschen und $60 \%$ der Westdeutschen auf eine Frage hin für schärferes Durchgreifen aus, bei der - in einer etwas überakzentuierten Polarisierung - zwischen der Option schärferes Durchgreifen und persönliche Freiheitsrechte - zu entscheiden war. Eine ein Jahr später durchgeführte Erhebung erbringt ähnliche Werte ( $68 \%$ vs. $84 \%$ ) (vgl. Noelle-Neumann und Köcher 1993:602; Noelle-Neumann und Köcher 1997:760).

3 Unter denen, die sich bedroht fühlen, plädieren $63 \%$ der Westdeutschen und $75 \%$ der Ostdeutschen für schärfere Gesetze. Unter denen, die sich nicht bedroht fühlen, liegen die Anteile bei $42 \%$ (West) und $64 \%$ (Ost) (unveröffentlichte Tabellen des Instituts für Demoskopie). Inwieweit das Sicherheitsgefühl punitives Denken begünstigt, kann mit diesen Angaben allein nicht geklärt werden, da Furcht über die sozialen Gruppierungen unterschiedlich verteilt ist und Drittvariablen die Beziehungen beeinflussen können.

4 Unter denen, die mehr als 3 Stunden fernsehen, plädieren $57 \%$ der Westdeutschen und $75 \%$ der Ostdeutschen für schärfere Gesetze. Unter denen, die weniger als 3 Stunden sehen, liegen die Zahlen bei $46 \%$ (West) und 64\% (Ost) (unveröffentlichte Tabelle des Instituts für Demoskopie). Für den Effekt des Fernsehens auf die Punitivität gilt im Übrigen, was auch schon über den Effekt der Kriminalitätsfurcht gesagt wurde (vgl. vorhergehende Anmerkung): eine direkte Messung dieses Effekts wäre nur bei Drittvariablenkontrolle möglich. 